\title{
A UV Flare at the Center of the Elliptical Galaxy NGC 4552
}

Lucio M. Buson ${ }^{1}$, Francesco Bertola ${ }^{2}$, David Burstein ${ }^{3}$, Michele Cappellari $^{2}$, Sperello di Serego Alighieri ${ }^{4}$, Laura Greggio ${ }^{5,6}$ \& Alvio Renzini $^{7}$

\begin{abstract}
A self-consistent analysis of near-UV, HST/FOC images of the elliptical galaxy NGC 4552 is used to show that its central spike has brightened by a factor $\sim 4.5$ between 1991 and 1993, and has decreased its luminosity by a factor $\sim 2.0$ between 1993 and 1996. A strong UV continuum over the energy distribution of the underlying galaxy is concurrently revealed shortward of $\lambda \sim 3200 \AA$ by our FOS spectra extending from the near-UV to red wavelengths. Nuclear emission-line profiles of both permitted and forbidden lines are best modelled with a combination of broad and narrow components, with FWHM of $\sim 3000 \mathrm{~km} \mathrm{~s}^{-1}$ and $\sim 700 \mathrm{~km} \mathrm{~s}^{-1}$, respectively. Current diagnostics based on the emission line intensity ratios definitely places the spike among AGNs, just at the border between Seyferts and LINERs. This evidence argues for the variable central spike being produced by a modest accretion event onto a central massive black hole (BH), with the accreted material having possibly being stripped from a star in a close fly-by with the BH. In this regard, one has to look at NGC 4552 as the faintest known AGN.
\end{abstract}

\section{The Serendipitous Discovery}

With the intent of studying the stellar populations of early-type galaxies, in 1993 we obtained FOC images in several ultraviolet bands of the central regions of a few nearby ellipticals galaxies, including NGC 4552 . The latter is a rather typical giant elliptical in the Virgo cluster whose absolute magnitude $\left(M_{\mathrm{B}}=-20.2\right.$, adopting a distance of $15.3 \mathrm{Mpc}$ ), structure, stellar population and metal content appear quite normal. At the time of our observation a point-like source with a photometric profile indistinguishable from the PSF of the aberrated HST

\footnotetext{
${ }^{1}$ Osservatorio di Capodimonte, Napoli, Italy

${ }^{2}$ Dipartimento di Astronomia, Università di Padova, Padova, Italy

${ }^{3}$ Department of Physics \& Astronomy, Arizona State University, Tempe, AZ, USA

${ }^{4}$ Osservatorio di Arcetri, Firenze, Italy

${ }^{5}$ Dipartimento di Astronomia, Università di Bologna, Bologna, Italy

${ }^{6}$ Sternwarte der Universität München, München, Germany

${ }^{7}$ European Southern Observatory, Garching bei München, Germany
} 
was present at its center (Bertola et al. 1995). In principle this is not surprising, as Maoz et al. (1996) show that $\sim 10 \%$ of nearby galaxy cores sampled in the ultraviolet with the FOC, do contain a unresolved point source in their centers. FOS spectra reveal, in turn, that UV-bright sources of this kind can be simply sub-parsec size star clusters (as in the case of NGC 2681; Cappellari et al. 1998a) or a centrally located starburst (Koraktar, this meeting).

The case of NGC 4552 turned out to be quite odd, however. By comparing our UV observation with an identical, pre-existing FOC image of this galaxy secured in 1991, we soon realized that we had detected its central spike just at a time it had increased its luminosity by a factor $\sim 7 \pm 1.5$ between the two epochs - as roughly estimated from aberrated data. The estimated luminosity of the UV-bright source in 1993 was $\sim 10^{6} \mathrm{~L}_{\odot}$ (Renzini et al. 1995). To get further clues to this phenomenon we obtained additional HST observations, including both UV/optical spectroscopy with FOS and FOC UV imaging, in 1996. These last observations confirm the variability of the central source (in the sense it is presently fading). So, though providing quite a loose sampling of the phenomenon, the existing FOC photometry strongly suggests that the spike which became visible at the center of the Virgo Elliptical NGC 4552 is a single, protracted UV flare caught in mid-action (see Fig. 1). This conclusion was made possible by fitting to the observed images a properly PSF-convolved galaxy model, consisting of an underlying constant nuker-law profile and a central, variable excess representing the spike. Such a procedure takes into account all needed correction factors for sensitivity differences and possible non-linearity effects of each FOC image, thus providing a self-consistent recalibration of the whole set of data (see Cappellari et al. 1998b for details).

With a bit of handsight it is quite clear one could have traced several hints of secondary events in the galaxy history as well as of BH-related nuclear activity well before our detection of the time-variable spike. Major signatures include extended $\mathrm{H} \alpha$ emission from the galaxy center out to $\sim 2.5 \mathrm{kpc}-$ likely the result of a recent accretion of a small gas rich satellite - (e.g. Macchetto et al. 1996), together with inner dust patches first recognized by van Dokkum \& Franx (1995) on early WFPC images, which turned out to form a circumnuclear, dusty ringlike structure in more detailed WFPC2 optical frames. Our own subtraction of more recent archive WFPC2 images does show this inner dusty feature in its full shape, i.e. as a complete ring encircling the central bright spike (Fig. 2).

\section{The UV-Dominated Continuum Spectrum of the Spike}

The availability of FOS, subarcsec UV/optical spectra of the flaring central spike proved to be an unprecedented source of physical insight onto the nature of this phenomenon, too. In Fig. 3 we compare the merged FOS spectra of the central $0^{\prime \prime} 21 \times 0.21$ region of NGC 4552 with a composite UV/optical spectrum meant to represent the underlying spectrum of the inner giant elliptical. Having normalized at the $\mathrm{V}$ band, we see that this composite spectrum is a good match in overall spectral energy distribution for the FOS spectrum, with two notable exceptions. Unlike the underlying spectrum the spike shows strong emission lines and, what is more, a remarkable continuum UV excess shortward of $\lambda 3200 \AA$. As far as the intrinsic UV SED of the spike is concerned, both the galaxy-subtracted 


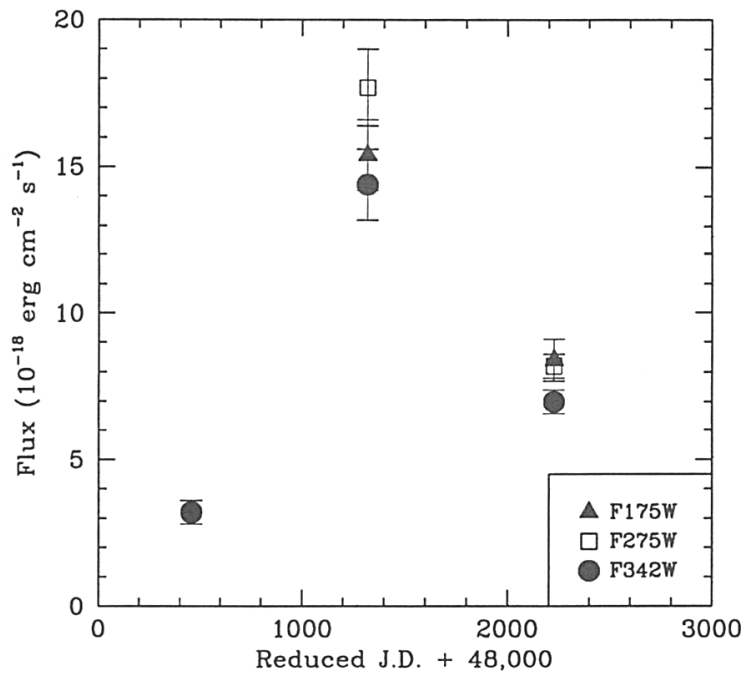

Figure 1. The light curve of the spike in the F175W, F275W and F342W passbands. The three epochs are July 1991, November 1993 and May 1996, respectively.

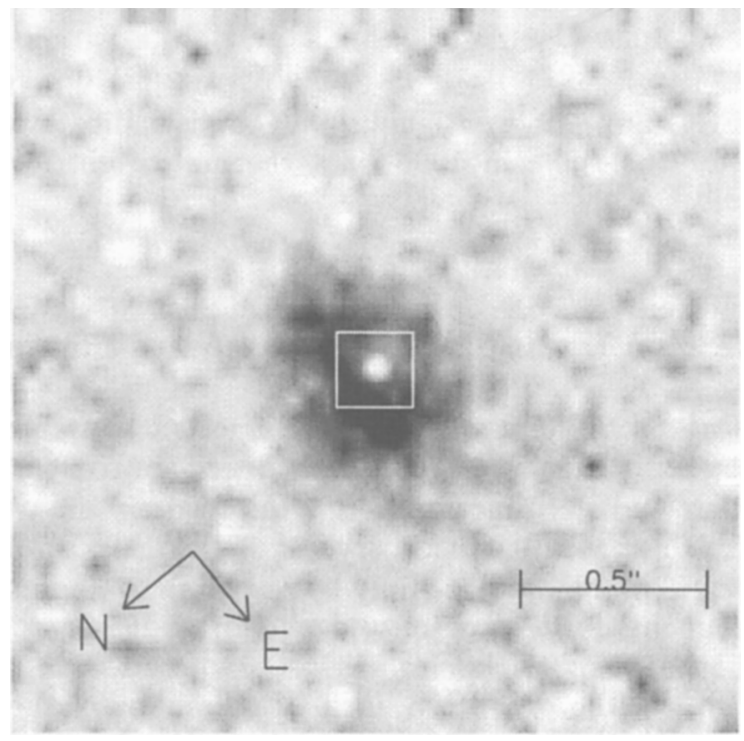

Figure 2. The $V-I$ (F555W-F814W) map of the central regions of NGC 4552. The darkest color correspond to $V-I \simeq 1.44 \mathrm{mag}$, while the background is at the $V-I \simeq 1.34 \mathrm{mag}$ level. The central square represents the size of the $0.2 \times 00^{\prime \prime} 2$ FOS aperture used for the spectroscopic observations. The central spike is clearly visible as a bright spot. 


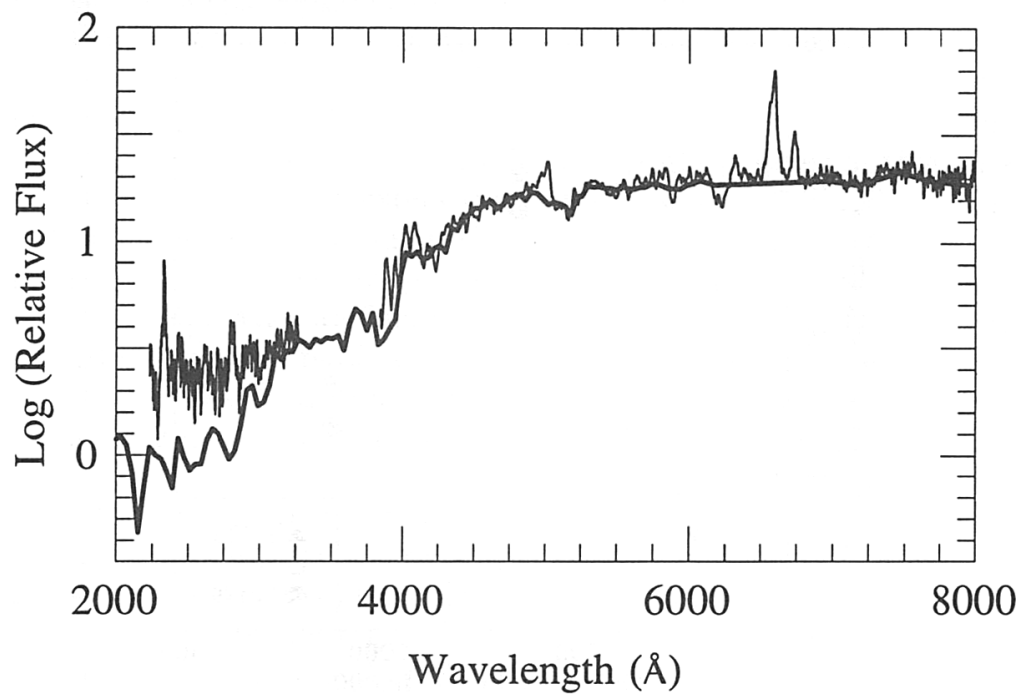

Figure 3. The overall 1996 FOS central spectrum of NGC 4552 within the $0 ! 2 \times 00^{\prime \prime} .2$ (thin line) superimposed to a scaled combination of the IUE $10^{\prime \prime} \times 20^{\prime \prime}$ aperture spectrum of NGC 4552 and a ground-based optical spectrum of the virtually identical giant elliptical NGC 4649. The spectra have been normalized to the visual region.

FOS SED and FOC fluxes in contiguous UV bands indicate a temperature of $T \sim 15,000 \mathrm{~K}$ for the spike in 1996 (if a thermal origin for the UV flux is assumed).

\section{The Uncommon Emission-Line Spectrum of the Spike}

The continuum-subtracted emission-line FOS spectra have been modeled as a whole by applying the Levenberg-Marquardt algorithm to fit a non-linear function. The resulting emission line fits permit us to draw many (some of which quite unusual) conclusions: [i] satisfactory fits of the emission lines identified in nuclear FOS spectra can be obtained only by resorting to a combination of broad and narrow components for both permitted and forbidden lines. This result is at variance with the behavior of classical AGNs - where the broad component is usually present only in the permitted lines [ii] the shape of the $\mathrm{H} \alpha+[\mathrm{NII}] \mathrm{com}$ plex has changed from the May 1996 spectrum to the January 1997 spectrum. In the context of our procedure, a satisfactory fit can be formally achieved only allowing for a shift to the blue of $\sim 230 \mathrm{~km} \mathrm{~s}^{-1}$ of the whole (narrow + broad) $\mathrm{H} \alpha$ line in the latter spectrum [iii] the ratios of the narrow emission line components of the spike spectrum place this feature definitely within the AGN region, just at the borderline between Seyferts and LINERs. Therefore, the spike in NGC 4552 can be either classified as a very high excitation LINER or a very low excitation Seyfert. 


\section{Towards a Self-consistent Interpretation}

The phenomenology of the spike in NGC 4552 is consistent with a scenario in which a central, UV-bright flare is caused by the tidal stripping of a star in a close flyby with a central supermassive $\mathrm{BH}$. Other interpretations (a central supernova, a central starburst, ...) were already considered unlikely. Circumstantial evidence in favor of the tidal stripping option comes now also from the noticed shift in the broad $\mathrm{H} \alpha$ emission between May 1996 and January 1997. Tidal stripping/disruption is indeed predicted to give rise to an elliptical accretion disk, the precession of which results in sizable $\mathrm{H} \alpha$ line profile variations. The stripping-induced flare is predicted to be very bright $\left(\sim 10^{10} \mathrm{~L}_{\odot}\right)$ for several years, much brighter than our observed flare, however. This indicates that if the flare in NGC 4552 was indeed caused by a tidal stripping in a BH-star flyby, it led to only partial stripping. As far as the broad forbidden lines of the NGC 4552 nucleus are concerned, taking into account we are dealing with a very low luminosity AGN, one can expect the forbidden lines to originate from the low-density disk itself, rather than from the usual distinct region farther away. If the whole emission line radiation does originate from a (thin) disk, then the gradient in rotational velocity should be responsible for the actual line profile, with the broadest part of it originating in the inner regions. Adopting the above scenario, the combined availability of high-resolution imaging and sub-arcsec spectroscopy allows us to put constraints to the mass of the central $\mathrm{BH}$. Our rough estimates, leading to a $\mathrm{BH}$ mass between $3 \times 10^{8}$ to $2 \times 10^{9} \mathrm{M}_{\odot}$, appear consistent with the mass of $M \simeq 4-6 \times 10^{8} \mathrm{M}_{\odot}$ of the supermassive central BH in NGC 4552, obtained by Magorrian et al. (1998), using simple isotropic dynamical models based on HST photometry and ground based kinematics.

\section{References}

Bertola, F., Cappellari, M., Burstein, D., Greggio, L., Renzini, A., di Serego Alighieri, S. 1995, Stellar Populations, ed. G. Gilmore \& P. van der Kruit (Dordrecht: Kluwer), p. 445

Cappellari, M., Bertola, F., Burstein, D., Buson, L.M., Greggio, L. \& Renzini, A.: UV Spikes in Bulge-Dominated Galaxies, 1998a, in: "STScI Workshop: How and When Do Bulges Form and Evolve?", C.M. Carollo, H.C. Ferguson \& R.F.G. Wyse (eds.), ASP Conf. Series, ASP, San Francisco

Cappellari, M., Renzini, A., Greggio, L., di Serego Alighieri, S., Buson, L.M., Burstein, D. \& Bertola, F. 1998b, ApJ, submitted

Macchetto, F., Pastoriza, M., Caon, N., Sparks, W.B., Giavalisco, M., Bender, R., \& Capaccioli, M. 1996, A\&AS, 120, 463

Magorrian, J., Tremaine, S., Richstone, D., Bender, R., Bower, G., Dressler, A., Faber, S.M., Gebhardt, K., et al. 1998, AJ, 115, 2285

Maoz, D., Filippenko, A.V., Ho, L.C., Macchetto, F.D., Rix, H.-W., \& Schneider, D.P. 1996, ApJS, 107, 215

Renzini, A., Greggio, L., Di Serego Alighieri, S., Cappellari, M., Burstein, D., \& Bertola, F. 1995, Nature, 378, 39

Van Dokkum, P.G. \& Franx, M. 1995, AJ, 110, 2027 